\title{
Contract research organizations on the Web
}

\section{Michael Francisco}

In the late 1990s, conventional wisdom holds that to become and stay successful, businesses need to have a presence on the Web. This is paticularly important for service companies, a category that includes contract research organizations (CROs). But exactly what kind of Web presence is needed, and what kind of information and features should CROs offer? Presumably, the information needed to make potential clients into actual ones. CROs have reaped the benefits as pharmaceutical companies compete to bring biotechnology-developed drugs to the marketplace: The cost of bringing a drug from development to commercialization is estimated at $\$ 500$ million', and CROs have carved niches for themselves at every stage of the drug development pipeline.

With a few exceptions, the Web sites of the leading CROs reviewed below seem to have followed the tried and true protocol for Web site content: company background, services offered, press releases, and contact information are all available. As is to be expected, quality in both content and design is highly variable.

Increasingly, however, CROs have been offering more on their Web sites. Supplemental content, as well as more sophisticated design and layout show that
CROs are also trying to attract job seekers, potential investors, and the just-plain-curious to visit their sites. Employment opportunities and investor information are becoming commonplace, and some companies offer unique features, such as clinical-study recruitment notices and opportunities for physicians interested in participation in clinical studies, that distinguish their sites from those of other companies and increase traffic. The following list covers Web sites for some leading contract research organizations.

1. Mossinghoff, G.J. 1995. Drug Info. J. 29:1077-1090.

\section{Web sites for selected contract research organizations}

\begin{abstract}
Pharmaceutical Product Development Inc.
http://www.ppdi.com/homepage.htm

PPD has an average site, with the standard corporate profile, recent press releases, investor information via a link to Nasdaq, and e-mail feedback capability.
\end{abstract}

\section{MDS Harris}

http://www.mdsharris.com/main.htm

This extensive site offers recent and archived press releases, a history of the company, complete contact information, and career opportunities. Also helpful are comprehensive study-recruitment information for anyone interested in volunteering in clinical trials, illustrated maps and schematics of services offered, and an alphabetized table of tested compounds and validated methods.

\section{Covance Inc.}

http://www.covance.com/

Covance's site has a useful listing of services available, career opportunities, investor information with links to financial sites, press releases, a site map, and e-mail feedback capability.

\section{Oread Inc.}

http://www.oread.com/

This site has a comprehensive description of Oread's divisions, capability, and operations, from preclinical development to manufacturing for commercial distribution. There is also an easy-to-read list of employment opportunities available. The site's unique feature is an online form for sending in such instruments as meters and gauges to Oread for calibration.

\section{Kendle International Inc.}

http://www.kendle.com/

Kendle's site is friendly, dotted with employee pictures and client testimonials, while also offering the usual features such as service information, industry news, employment opportinities, press releases, and investor information. The layout is simple and clear, and a notable feature is an e-mail form for physicians interested in paticipating in Kendle studies.

\section{MDS Panlabs}

\section{www.panlabs.com}

Another easy-to-use average site, offering background on the company and services offered. A PowerPoint presentation is available for downloading.

\section{Quintiles Transnational Corp.}

http://www.cro.quintiles.com/

Quintiles' site has extensive information on the company's data management products and services, a feedback form, a page of selected links to pharmaceutical, data management, and clinical trials resources, complete contact information for Quintiles worldwide, and up-to-date press releases.

\section{ClinTrials Research}

http://www.clintrials.com/aboutctr.htm

ClinTrials' site suffers from lack of text-editing (two typographical errors were noted). It offers the same standard features as other sites, but without many graphics. The employment opprtunities page has a biographical data feedback form for the company's personnel files. 